\title{
Tracing the evolution within nearby galaxy groups: a multi-wavelength approach
}

\author{
Daniela Bettoni $^{1}$, Antonina Marino ${ }^{2}$, Roberto Rampazzo ${ }^{1}$, \\ Henri Plana ${ }^{3}$, Margarita Rosado ${ }^{4}$, Giuseppe Galletta ${ }^{2}$, Paola Mazzei ${ }^{1}$, \\ Luciana Bianchi ${ }^{5}$, Lucio M. Buson ${ }^{1}$, Patricia Ambrocio-Cruz ${ }^{6}$ and \\ Ruslan Gabbasov ${ }^{4}$ \\ ${ }^{1}$ Inaf - Osservatorio Astronomico di Padova, Italy \\ email: daniela.bettoni@oapd.inaf.it \\ ${ }^{2}$ Dept. of Physics \& Astronomy University of Padova, Italy \\ email: antonina.marino@unipd.it \\ ${ }^{3}$ Laboratorio de Astrofisica Teorica e Observacional, Universidade de Santa Cruz, Brasil \\ ${ }^{4}$ Instituto de Astronomia Universidad National Autonoma de Mexico, Mexico \\ ${ }^{5}$ Dept of physics and Astronomy, Johns Hopkins University, Baltimore, USA \\ ${ }^{6}$ Universidad Autonoma del Estado de Hidalgo, area academica de Ciencias de la Tierra y \\ Materiales, Mexico
}

\begin{abstract}
Evolutionary scenarios suggest that several mechanisms (from inner secular evolution to accretion/merging) may transform galaxy members, driving groups from an active star forming phase to a more passive, typical of dense environments. We are investigating this transition in a nearby group sample, designed to cover a wide range of properties (see also Marino et al. (2010), Bettoni et al. (2011) and Marino et al. (2012)). We study two groups, USGC U268 and USGC U376 located in different regions of the Leo cloud, through a photometric and kinematic characterization of their member galaxies. We revisit the group membership, using results from recent red-shift surveys, and we investigate their substructures. U268, composed of 10 catalogued members and 11 new added members, has a small fraction $(\sim 24 \%)$ of early-type galaxies (ETGs). U376 has 16 plus 8 new added members, with $\sim 38 \%$ of ETGs. We find the significant substructuring in both groups suggesting that they are likely accreting galaxies. U268 is located in a more loose environment than U376. For each member galaxy, broad band integrated and surface photometry have been obtained in far-UV (FUV) and near-UV (NUV) with GALEX, and in $u, g, r, i, z$ (SDSS) bands. $\mathrm{H}_{\alpha}$ imaging and $2 \mathrm{D}$ high resolution kinematical data have been obtained using PUMA Scanning Fabry-Perot interferometer at the $2.12 \mathrm{~m}$ telescope in San Pedro Mártir (Baja California, México). We improved the galaxy classification and we detected morphological and kinematical distortions that may be connected to either on-going and/or past interaction/accretion events or environmental induced secular evolution. U268 appears more active than U376, with a large fraction of galaxies showing interaction signatures (60\% vs. 13\%). The presence of bars among late-type galaxies is 10\% in U268 and $29 \%$ in U376. The cumulative distribution of (FUV - NUV) colors of galaxies in U268 is significantly different (bluer) than that of U376's galaxies. Most (80\%) of the early-type members in U376 inhabits the red sequence, a large fraction of galaxies, of different morphological types, are located in the green valley, while the blue sequence is under-populated with respect to U268.
\end{abstract}

Keywords. galaxies: evolution, galaxies: groups, galaxies: photometry, ultraviolet: galaxies

\section{References}

Bettoni, D., Galletta, G., Rampazzo, R., Marino, A., Mazzei, P., \& Buson L. 2011, A $ॄ$ A, 534, A24

Marino A., Bianchi L., Rampazzo R., Buson L. M., \& Bettoni D. 2010, A\&4A, 511, A29

Marino A., Plana, H., Rampazzo R., et al. 2012, MNRAS, in press. arXiv:1209.4278 